\title{
Enfoque de la diarrea en pacientes infectados con VIH
}

\section{Approach to diarrhea in HIV patients}

\author{
Álvaro Andrés Gómez Venegas, ${ }^{1}$ Luis Alfredo Moreno Castaño, ${ }^{2}$ Jairo Alonso Roa Chaparro. ${ }^{3}$
}

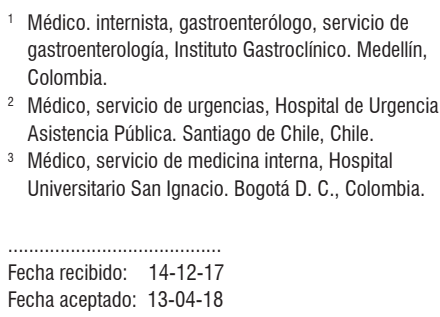
gastroenterología, Instituto Gastroclínico. Medellín, Colombia.

2 Médico, servicio de urgencias, Hospital de Urgencia Asistencia Pública. Santiago de Chile, Chile.

${ }^{3}$ Médico, servicio de medicina interna, Hospital Universitario San Ignacio. Bogotá D. C., Colombia.

Fecha recibido: $14-12-17$ Fecha aceptado: 13-04-18

\begin{abstract}
Resumen
La diarrea es el síntoma gastrointestinal más frecuente en las personas infectadas por el virus de la inmunodeficiencia humana $(\mathrm{VIH})$. La diarrea puede aparecer como consecuencia de infección por un germen oportunista, así como ser un efecto secundario del tratamiento antirretroviral. Esta, a su vez, puede ser aguda o crónica, esta última es la que causa mayor morbilidad y alteración en la calidad de vida del paciente. El enfoque diagnóstico se realiza por etapas que van desde una historia clínica completa hasta estudios microbiológicos, endoscópicos e imagenológicos. Finalmente, si se han descartado causas infecciosas u orgánicas (enteropatía idiopática), se debe brindar manejo al paciente en busca de aliviar los síntomas y optimizar la adherencia al tratamiento antirretroviral.
\end{abstract}

\section{Palabras clave}

Diarrea, virus de inmunodeficiencia humana, $\mathrm{VIH}$, antirretrovirales, enteropatía por $\mathrm{VIH}$.

\section{Abstract}

Diarrhea is the most common gastrointestinal symptom in people with human immunodeficiency virus infections. Diarrhea can appear to be a consequence of infection by an opportunistic germ or the side effect of antiretroviral treatment. It can be acute or chronic, but the latter leads to greater morbidity and alteration in patients' quality of life. Stages of the diagnostic approach range from taking a complete clinical history, to microbiological, endoscopic and imaging studies. Finally, if infectious or organic causes have been ruled out (idiopathic enteropathy), management provided to the patient should seek symptomatic relief and optimization of adherence to antiretroviral treatment.

\section{Keywords}

Diarrhea, human immunodeficiency virus, HIV antiretrovirals HIV enteropathy.

\section{INTRODUCCIÓN}

La diarrea es el síntoma gastrointestinal más frecuente en las personas infectadas por el virus de la inmunodeficiencia humana (VIH) (1). Hasta un $40 \%-80 \%$ de pacientes con VIH sin tratamiento llega a presentar diarrea $(2,3)$ $y$ puede aparecer por el uso de antimicrobianos o como efecto secundario de la terapia antirretroviral (TARV) (1).
La diarrea durante más de 1 mes junto con pérdida de peso es una condición incluida en la definición del síndrome de inmunodeficiencia adquirida (SIDA) (1).

En Colombia, pocos estudios han evaluado la diarrea en pacientes con VIH. Un estudio en Medellín con 159 pacientes hospitalizados encontró que los síntomas gastrointestinales se presentaron en un 50,3\% y la definición de SIDA por diarrea crónica en $4,7 \%$ de los casos. Se diagnosticaron 
$33 \%$ de infecciones oportunistas: tuberculosis (37\%), histoplasmosis (17\%) y criptococosis (9,7\%) (4). Otro estudio colombiano en 115 pacientes con diarrea encontró una infección por Cryptosporidium en 10,4\% y 29\% de positividad por microsporidios con cromotropo modificada. La prevalencia de parásitos fue de 59,1\% (Blastocystis hominis: $25,2 \%$ y Entamoeba histolytica: $13 \%$ ) (5). Un trabajo realizado en India encontró predominio de parásitos en comparación con bacterias y hongos en pacientes con diarrea $(58,3 \%$ frente a $29,17 \%$ y $12,50 \%$, respectivamente). El parasito más común fue Isospora $(25,9 \%)$ y la bacteria más común fue Escherichia coli enterotoxigénica (18,5\%) con algunos casos de Shigella y Mycobacterium tuberculosis (3,7\% cada uno) (6).

\section{FISIOPATOLOGÍA}

El sistema inmune de las mucosas del tracto digestivo (GALT) cumple las funciones de barrera y protección, discrimina antígenos alergénicos o patogénicos y promueve la eliminación o la tolerancia a los mismos. Hacen parte del GALT los linfocitos intraepiteliales, que se ubican en la membrana basal del epitelio intestinal entre los enterocitos. La mayoría son linfocitos $\mathrm{T}$ de los fenotipos CD3, CD4 y CD8; y son los encargados de regular en conjunto con linfocitos B las funciones ya descritas de tolerancia o eliminación de antígenos (2). Una vez el VIH penetra a través o entre las células epiteliales del aparato digestivo, se deposita a través de los receptores CCR5 de las células M en el "bolsillo basal", donde tiene contacto con linfocitos de la lámina propia, que son las células blanco del virus, lo que causa la apoptosis y posterior disminución del número de estas células, inicialmente a nivel epitelial y luego a nivel del sistema linfático (1-3).

El ácido ribonucleico (ARN) del VIH se ha identificado en el intestino del $66 \%$ de pacientes con diarrea, en comparación con el $45 \%$ de pacientes sin diarrea (7). Por este tipo de estudios se ha planteado la posibilidad de un daño citopático directo por el VIH al enterocito, mediado al parecer directamente por la glucoproteína 120 (gp120) y así poder generar la denominada enteropatía idiopática asociada a VIH, en la cual se ha descartado una infección por gérmenes oportunistas.

En las células epiteliales intestinales se han descrito alteraciones en el citoesqueleto con uniones intercelulares débiles, lo que genera mayor permeabilidad y, por consiguiente, pérdida de líquidos y electrólitos. También se han descrito atrofia de vellosidades, hiperplasia de criptas y disminución de disacaridasas que, en conjunto con algún grado de disfunción funcional ileal, promueven la malabsorción de carbohidratos, sales biliares y vitaminas. Otras proteínas implicadas en la lesión directa por el VIH inclu- yen la proteína Tat I, que induce la secreción de cloro e inhibe la proliferación del enterocito; además de la proteína $\mathrm{R}$, que promueve la formación de radicales libres (1-3).

Otro fenómeno pocas veces estudiado en la enteropatía idiopática por VIH es la insuficiencia pancreática exocrina (no relacionada con didanosina), que podría empeorar la malabsorción de nutrientes. La coinfección por hepatitis $\mathrm{C}$ (VHC) con tratamiento y el uso de alcohol parecen ser factores asociados con dicha alteración pancreática, que podría explicarse inclusive por la generación de autoanticuerpos contra la glándula (1-3). También se ha descrito la neuropatía autonómica por daño nervioso directo por el VIH (1-3). En especial, los cambios citopáticos en el intestino delgado tienden a mejorar con la TARV, confirmando el daño directo al enterocito y el sistema inmune del aparato digestivo.

\section{CAUSAS INFECCIOSAS}

Es importante tener en cuenta que hasta en un 50\% de pacientes con infección por VIH y diarrea no se logra el aislamiento de gérmenes patógenos. De todas maneras, siempre se deben realizar estudios en búsqueda de agentes microbiológicos debido a que el pronóstico y desenlace del paciente se modifica de manera importante en caso de lograr un diagnóstico puntual. Es una amplia lista la que se ha descrito de potenciales gérmenes asociados con diarrea en VIH. A continuación, se revisan brevemente los más relevantes en nuestro medio.

\section{Infecciones por bacterias}

Los pacientes con VIH tienen un riesgo similar de desarrollar diarrea por bacterias que los pacientes inmunocompetentes, aunque en los primeros se presenta con mayor compromiso sistémico $(2,8)$. La infección por Campilobacter cursa con diarrea, dolor abdominal, fiebre e inclusive bacteriemia (9). El diagnóstico se realiza con coprocultivo. La infección por Salmonella no tifoidea origina en el paciente con VIH gastroenteritis, bacteriemia e infección local o diseminada (10). El diagnóstico se puede realizar mediante coprocultivo o hemocultivos.

El manejo de estas infecciones se basa en ciprofloxacina, que se recomienda por 14 días. En pacientes con proctocolitis y VIH, se debería sospechar infección por Chlamydia trachomatis. El diagnóstico se realiza con coprocultivo y hemocultivo dada la alta prevalencia de bacteriemia en inmunosuprimidos (11). Se sugiere tratar con doxiciclina (100 mg cada 12 horas por 7 días) o azitromicina ( $1 \mathrm{~g}$, dosis única).

Aunque no es muy frecuente, las infecciones por micobacterias pueden comprometer el aparato digestivo, siendo el grupo de Mycobacterium avium complex (MAC) la más 
común $(12,13)$. La enfermedad diseminada por la MAC era la infección bacteriana oportunista más común en pacientes con SIDA. Con el advenimiento de la TARV, su incidencia ha declinado y se presenta en pacientes con mal control inmunovirológico. La presentación clínica es variable con fiebre, pérdida de peso, diaforesis nocturna, diarrea acuosa, malabsorción, linfadenopatías y megalias (14). Usualmente, compromete el duodeno y se sospecha al evidenciar en la esofagogastroduodenoscopia (EGD) nódulos mucosos o parches amarillentos (15). En la biopsia se pueden observar macrófagos con inclusiones de bacilos ácido-rápido similares a los presentados en la Enfermedad de Whipple. También se puede diagnosticar en hemocultivos y coprocultivos (14). Se sugiere manejar con claritromicina (500 mg vía oral [VO] cada 12 horas) y etambutol ( $15 \mathrm{mg} / \mathrm{kg} / \mathrm{una}$ vez al día VO) con o sin rifabutina (300 mg una vez a día) (16).

Clostridium difficile es una de las bacterias asociadas con diarrea en pacientes con VIH. El diagnóstico se puede establecer mediante técnicas de inmunoensayo que detectan toxinas A y B, con sensibilidad del 70\%-78\%. El estudio de antígeno común aporta mayor sensibilidad, aunque no discrimina entre las cepas patógenas y las no patógenas (17).

La técnica de reacción en cadena de la polimerasa (PCR), que amplifica genes para toxinas A y B, ofrece mayor sensibilidad y especificidad en comparación con las demás técnicas (18). La prueba de oro para el diagnóstico es el cultivo, con la limitante de que puede tardar hasta 72 horas. El manejo estándar consiste en metronidazol $500 \mathrm{mg}$ VO cada 8 horas por 10-14 días o vancomicina oral $125-250 \mathrm{mg}$ cada 6 horas por 10-14 días.

En infecciones severas, por cepas virulentas, o en pacientes con VIH, la vancomicina pareciera superior al metronidazol. Otra alternativa es la fidaxomicina, antibiótico no absorbible en el tracto gastrointestinal (200 mg cada 12 horas por 10 días) que posee una eficacia cercana a la vancomicina (19).

\section{Infecciones por virus}

En pacientes con VIH, el citomegalovirus (CMV) genera altas tasas de morbimortalidad y puede afectar cualquier porción del tracto gastrointestinal. Se manifiesta con fiebre, pérdida de peso, anorexia, dolor abdominal y diarrea sanguinolenta. La colonoscopia es el método diagnóstico de elección que evidencia en la mucosa eritema en parches, erosiones y úlceras $(20,21)$.

El antiviral más efectivo es el ganciclovir $(5 \mathrm{mg} / \mathrm{kg}$ intravenoso [IV] cada 12 horas por al menos 3 semanas). Como alternativa, se encuentran el valganciclovir $(900 \mathrm{mg}$ cada 12 horas $\mathrm{VO}$ por al menos 3 semanas) y foscarnet ( $90 \mathrm{mg}$ intravenoso cada 12 horas de 3 a 6 semanas).

\section{Infecciones por parásitos}

Los parásitos que causan diarrea en pacientes con VIH incluyen aquellos que también pueden generar infección en inmunocompetentes como Giardia lamblia, Entamoeba histolitica y Estrongiloides stercoralis. Adicionalmente, existen parásitos oportunistas que no generan enfermedades en la población sana.

La infección por Criptosporidium compromete el intestino delgado, lo que genera diarrea severa en pacientes con VIH. También tiene la facultad de infectar el epitelio de las vías respiratorias y la vía biliar $(22,23)$. Con la llegada de la TARV ha disminuido la morbilidad atribuida al Criptosporidium $(24,25)$. El diagnóstico se realiza por Ziehl-Neelsen modificado en la materia fecal, en la que se identifican oocitos o PCR en biopsias de intestino delgado o recto (8). El tratamiento es la administración de TARV, cuyo objetivo es aumentar el recuento de linfocitos T CD4 (23). Existe alguna evidencia que sugiere manejo con nitazoxanida $500 \mathrm{mg}$ cada 12 horas hasta resolver los síntomas y lograr la erradicación en la materia fecal $(8,24)$.

La Isospora belli puede causar diarrea, vómito, dolor abdominal y pérdida de peso (25). Las opciones terapéuticas en este caso son trimetoprima/sulfametoxazol $(160 \mathrm{mg} / 800$ $\mathrm{mg}$ ) cada 6 horas por 10 días o ciprofloxacino $500 \mathrm{mg}$ cada 12 horas por 7 días en caso de alergia a las sulfas (8).

El espectro de infección por E. stercolaris en pacientes con VIH es variable: desde cuadros de diarrea crónica y anemia, hasta sangrado digestivo, obstrucción intestinal o inclusive síndrome de hiperinfección $(26,27)$.

El síndrome de hiperinfección predomina en pacientes adultos de género masculino y se caracteriza por multiplicación larvaria incontrolada, con un incremento importante en el número de larvas infectantes por fuera del aparato digestivo por medio de los espacios vasculares y alveolares, lo que genera edema pulmonar, neumonía y hemorragia alveolar, hasta evolucionar a insuficiencia respiratoria y multiorgánica, con una mortalidad hasta del $80 \%$. El diagnóstico se realiza mediante observación de las larvas a nivel pulmonar.

Otros sistemas comprometidos en la hiperinfección son el sistema nervioso central (SNC), cursando los pacientes con meningitis, diversas lesiones cutáneas que van desde la Larva currens hasta erupciones maculopapulares periumbilicales, púrpura y petequias. También se encuentra el hígado con obstrucción biliar o inflamación portal granulomatosa; y, menos común, el corazón, tiroides, páncreas y vejiga, entre otros $(26,27)$.

El diagnóstico se realiza usualmente por estudio directo en la materia fecal, aunque el método más sensible es la biopsia duodenal. Se recomienda tratar con ivermectina en dosis de $200 \mu \mathrm{g} / \mathrm{kg}$ VO una vez al día durante 1 o 2 días. De 
ser necesario, el tratamiento se puede repetir 2 a 3 semanas después de la primera dosis (27). En el síndrome de hiperinfección se deberá administrar ivermectina diariamente hasta que los síntomas resuelvan y las pruebas en la materia fecal sean negativas durante 2 semanas (27).

\section{Infecciones por hongos}

El compromiso gastrointestinal por Histoplasma capsulatum es infrecuente, ocurre en pacientes con niveles bajos de CD4 y afecta usualmente la región ileocecal. Para su diagnóstico se pueden realizar cultivos de materia fecal, hemocultivos, identificación de antígeno urinario y del espécimen en biopsia $(28,29)$. Se debe tratar con anfotericina B por 1-2 semanas seguido de itraconazol por 12 meses. En pacientes con VIH y conteo de CD4 $<150$ células $/ \mathrm{mm}^{3}$ que habiten en zonas endémicas, deberán recibir profilaxis con itraconazol $200 \mathrm{mg} /$ día (29).

Las manifestaciones de infección por Microsporidium incluyen diarrea crónica no inflamatoria, pérdida de peso, dolor abdominal, náuseas, vómito y fiebre (30). El diagnóstico del germen es un reto dado su pequeño tamaño ( 1 a $2 \mu \mathrm{m})$ y el patrón de oro para su diagnóstico sigue siendo la tinción Ziehl-Neelsen modificada; sin embargo, existen otros métodos como la PCR y el ensayo por inmunoabsorción ligado a enzimas (ELISA) (30). El tratamiento se basa en albendazol ( $400 \mathrm{mg}$ cada 12 horas por 3 semanas), pero la TARV con el aumento de CD4 tiende a resolver la infección (11).

\section{CAUSAS NO INFECCIOSAS}

La enteropatía idiopática por VIH se diagnostica una vez se haya descartado la infección por gérmenes patógenos y ocurre en un $50 \%$ de los pacientes (31). Se caracteriza por presentar diarrea más bien acuosa, que empeora con el consumo de alimentos y mejora con la deposición; síntomas similares a los descritos en el síndrome de intestino irritable (SII). Es necesario realizar una aclaración, ya que se puede caer en el error de diagnosticar tempranamente SII en pacientes con VIH y diarrea crónica, y se debe saber que dado los múltiples mecanismos fisiopatológicos descritos (algunos irreversibles a pesar del tratamiento), con alteración orgánica del tracto digestivo, prácticamente reducen este diagnóstico a un subgrupo de pacientes con VIH. Se sugiere reservar el diagnóstico de SII solamente para los pacientes con VIH que desde un principio en su enfermedad lograron tener un adecuado control inmunovirológico $y$ en quienes definitivamente se ha realizado un estudio exhaustivo que ha descartado una infección oportunista o alteración estructural del aparato digestivo (incluida la insuficiencia pancreática exocrina).
La enteropatía por VIH tiene un impacto negativo en la calidad de vida de los pacientes, evidenciado en múltiples estudios demográficos, en los que se ha encontrado, por ejemplo, que hasta en un 40\% esta llegó a afectar su vida social, razón por la cual se deberían agotar las opciones terapéuticas en busca de la mejoría sintomática.

También se ha descrito la diarrea como un efecto secundario de la TARV, lo que es importante por las altas tasas de abandono del tratamiento (32). Los inhibidores de la proteasa son los agentes con mayor asociación (33). Ritonavir es uno de los más reportados (hasta en 10\%-15\% de pacientes), en combinación con lopinavir y fosamprenavir, por lo que existen algunas combinaciones con menores tasas de diarrea como atazanavir-ritonavir, darunavir-ritonavir y saquinavir-ritonavir $(2,8)$.

Se han propuesto diversos mecanismos fisiopatológicos que explican la diarrea asociada con la TARV. Por ejemplo, se encontró que el nelfinavir podría estimular las vías de señalización $\mathrm{Cabb}$ en las células epiteliales, que causa pérdida de cloro a través de las membranas epiteliales. Lopinavir se ha relacionado con apoptosis celular y disrupción de la barrera intestinal, lo que inclusive causa erosiones de la mucosa en duodeno e íleon $(2,33)$. Las alteraciones funcionales y estructurales de los enterocitos en pacientes con inhibidores de proteasas producen un aumento en la concentración de electrólitos y del $\mathrm{pH}$ fecal, con un cambio en el gap osmótico y, por consiguiente, diarrea secretora.

\section{ENFOQUE DIAGNÓSTICO}

\section{Primera etapa: evaluación del compromiso por gérmenes (34)}

En primer lugar, debe realizarse una anamnesis completa que se enfoque en la evolución de la infección por VIH, manejo recibido, adherencia y factores de riesgo para infecciones como viaje a zonas endémicas (importante para amebas o giardias), relaciones sexuales por vía anal ( $C$. trachomatis o herpes) o uso reciente de antibióticos ( $C$. difficile). Se debe definir si la diarrea es aguda o crónica y categorizarla en 4 grados (35):

- Grado 1. Leve: diarrea transitoria o intermitente con menos de 3 deposiciones/día sobre el patrón normal.

- Grado 2. Moderada: diarrea líquida persistente o aumento de 4 a 6 deposiciones.

- Grado 3. Severa: diarrea sanguinolenta o más de 7 deposiciones/día que requieran manejo con líquidos IV.

- Grado 4. Potencialmente incompatible con la vida: con choque o disfunción de órganos.

Esta clasificación permite establecer el lugar de estudio del paciente (ambulatorio u hospitalario), prioridad de estu- 
dios, probabilidad de diagnosticar oportunistas y necesidad de iniciar el manejo empírico.

En el examen físico se debe evaluar el perfil nutricional y signos de deshidratación. Si existen síntomas oculares, se debe revisar el fondo del ojo en busca de retinitis por CMV o por Microsporidium. Se debe determinar la presencia de hepatoesplenomegalia. Si el paciente refiere dolor perianal, es necesario realizar tacto rectal para descartar infecciones de transmisión sexual (Figura 1).

Para mejorar el rendimiento en la detección de gérmenes, se deben tomar mínimo 3 muestras para coprológico en un período de 10 días (36). Siempre que haya riesgo para infección por Microsporidium, Criptosporidium o Isospora se debe realizar tinción con Ziehl-Neelsen modificado. Si existe sospecha de infección por CMV, se debe realizar un estudio de sangre para la medición del antígeno o inmunoglobulina $\mathrm{M}(\mathrm{IgM})$ y, de persistir la sospecha, métodos directos como PCR en sangre y materia fecal. Las muestras para cultivo de Shigella y Salmonella deben transportarse de inmediato al laboratorio dado que el cambio de $\mathrm{pH}$ sin refrigeración altera su rendimiento. Para la detección de $C$. difficile, inicialmente se debe estudiar con toxina A y B en la materia fecal. La probabilidad de cultivar micobacterias en materia fecal es baja, por tanto, no es recomendable. Determinar el nivel de CD4 es fundamental para orientar las causas de diarrea en pacientes con VIH $(1,3)$ (Tabla 1, Figura 2).

\section{Segunda etapa: examen del tracto gastrointestinal (34)}

Si en la primera fase no se diagnostica una infección por patógenos y la diarrea persiste y es severa, se deben realizar estudios endoscópicos o radiológicos $(34,37)$. Las guías sobre el estudio endoscópico de la diarrea de la American Society for Gastrointestinal Endoscopy (ASGE) (37) recomiendan realizar inicialmente sigmoidoscopia en pacientes con VIH, pero aclaran que, si la sigmoidoscopia no es positiva y la probabilidad de infección oportunista es alta, se debe realizar colonoscopia con biopsias de íleon y colon, y EGD con biopsias de duodeno (Figura 3).

Varios estudios han demostrado la utilidad de los procedimientos endoscópicos en este contexto. Se debe aclarar que no hay un patrón endoscópico típico de infección por oportunistas, por tanto, siempre se deben tomar biopsias. Se sugiere que la infección por Salmonella predomina en el colon derecho y va desde un eritema hasta ulceraciones. La infección por amebas suele afectar el ciego y rectosigmoide, con ulceraciones y zonas de necrosis (Figura 4). El CMV puede generar ulceraciones que predominan en el colon izquierdo (Figura 5). El rendimiento de la colonoscopia va desde el $27 \%$ al 39\%, y el CMV es el germen más común (38).

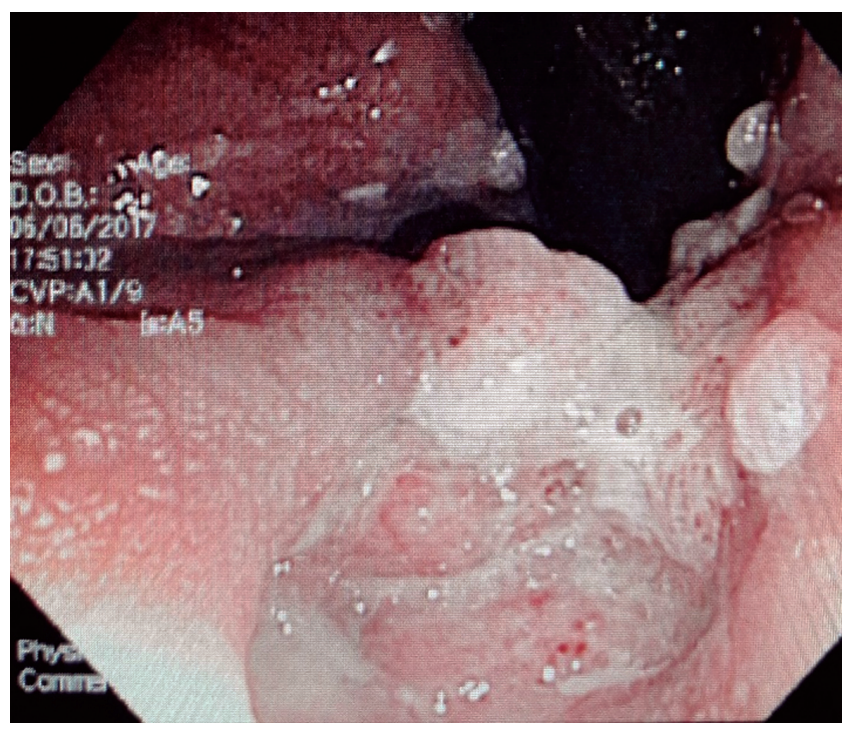

Figura 1. Sigmoidoscopia en paciente con VIH. Múltiples lesiones verrucosas en el recto que se extienden hacia el canal anal y son sugestivas de condilomas perianales.

Tabla 1. Nivel de CD4 y riesgo de infección por gérmenes asociados con diarrea en VIH

\begin{tabular}{ll}
\hline \multicolumn{1}{c}{ Conteo de CD4 } & \multicolumn{1}{c}{ Tipo de gérmenes asociados } \\
\hline Cualquier conteo de CD4 & $\begin{array}{l}\text { Salmonella, Campylobacter, Tuberculosis, } \\
\text { C. difficile, Giardia, Entamoeba, } \\
\text { Estrongiloides }\end{array}$ \\
& Criptosporidium \\
$<200$ células $/ \mathrm{mm}^{3}$ & Histoplasma \\
$<150$ células $/ \mathrm{mm}^{3}$ & Isospora, Microsporidium \\
$<100$ células $/ \mathrm{mm}^{3}$ & MAC, CMV \\
$<50$ células $/ \mathrm{mm}^{3}$ &
\end{tabular}

Un estudio comparó los exámenes microbiológicos en la materia fecal con las biopsias por endoscopia y se encontró que estos últimos tienen mayor rendimiento en pacientes con CD4 $<200$ células $/ \mathrm{mm}^{3}$ (39). Otro estudio diagnosticó una infección oportunista por métodos endoscópicos en 21/48 pacientes (44\%; intervalo de confianza [IC] 95\%: 30\%$58 \%)$. La colonoscopia encontró el diagnóstico en 13 pacientes, incluido el CMV en 9 de ellos. En la mayoría de pacientes se realizó el diagnóstico por biopsias del rectosigmoide. La EGD diagnosticó una infección por Microsporidium en 7 pacientes y Criptosporidium en 2 pacientes (40).

Otro trabajo prospectivo con 79 pacientes encontró el diagnóstico en 22 casos, siendo las biopsias de colon izquierdo las que mayor aportaron (17/22 pacientes con sensibilidad del $77 \%$ ) y en $15 / 15$ pacientes con infección 


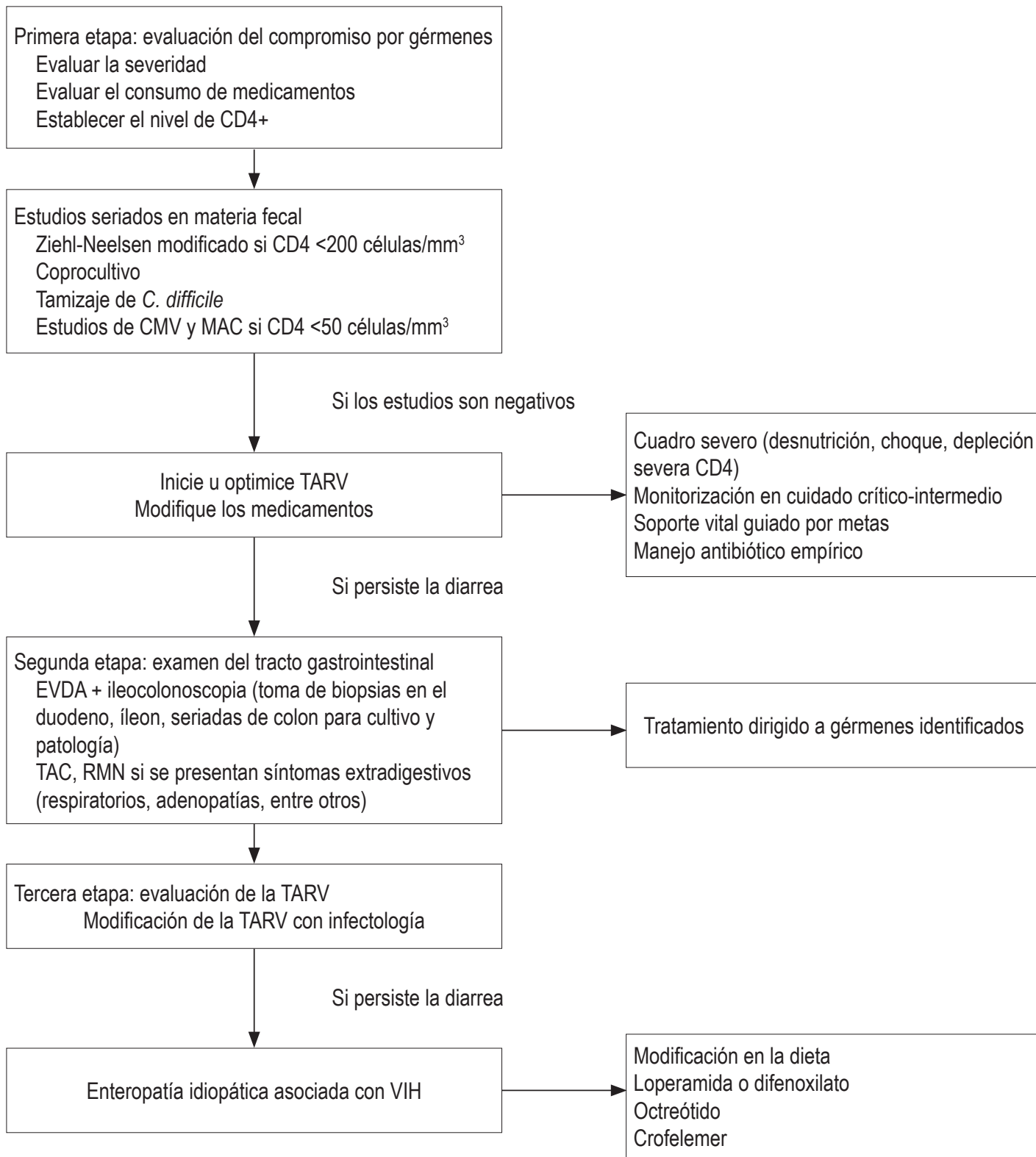

Figura 2. Flujograma del enfoque de la diarrea en pacientes con VIH. EVDA: endoscopia de vías digestivas altas; RMN: resonancia magnética nuclear; TAC: tomografía axial computarizada.

por CMV (sensibilidad del 100\%). La combinación de biopsias de colon izquierdo y derecho tuvieron sensibilidad del $82 \%$. Las biopsias de duodeno por EGD no aportaron al diagnóstico cuando se compararon con las tomadas por colonoscopia (41). Un estudio con 40 pacientes encontró el diagnóstico por colonoscopia en el $65 \%$, y la colitis amebiana y la CMV son las principales causas (42).
Siempre que se realicen procedimientos endoscópicos, independientemente de los hallazgos, se deben tomar biopsias tanto de colon como de intestino delgado. Aunque el rendimiento diagnóstico de las biopsias de mucosa sana es menor, se podrían diagnosticar infecciones oportunistas (43). La infección aislada por CMV en colon derecho llega a ser hasta del 29\%-39\%, por lo que la colonoscopia total es 


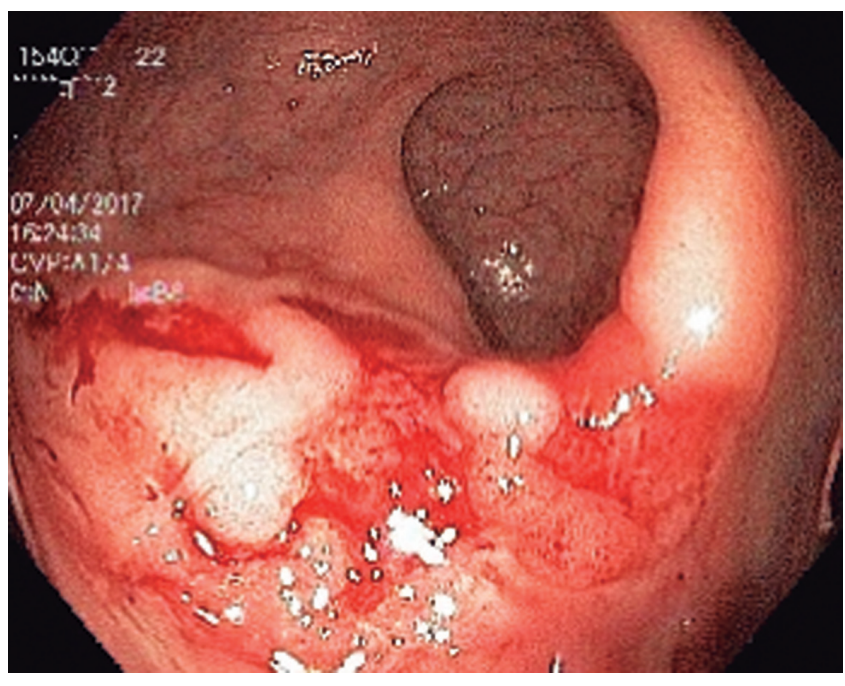

Figura 3. Rectoscopia de un paciente con VIH y relaciones sexuales por vía anal. Gran úlcera rectal por virus del herpes.

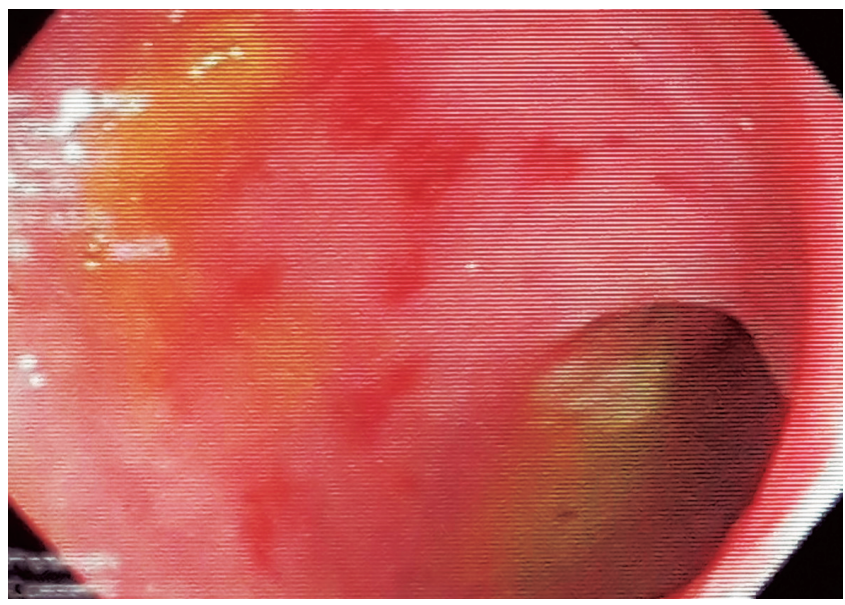

Figura 5. Sigmoidoscopia en un paciente con VIH y diarrea sanguinolenta. Se observan múltiples erosiones redondas en parches con inmunohistoquímica (IHQ) que sugieren una infección por CMV.

de elección sobre la sigmoidoscopia $(38,44)$. Si la sospecha es alta, deben realizarse PCR e IHQ a las biopsias. Siempre que se tomen biopsias para estudio microbiológico deben enviarse en tubo seco o en solución salina, jamás en formol.

Cuando se realice una EGD se deben tomar biopsias de duodeno lo más distales posibles (tercera y cuarta porción duodenal), ya que se aumenta la detección de Microsporidium. El aspirado duodenal no ha demostrado aumentar la detección de patógenos y no debería realizarse

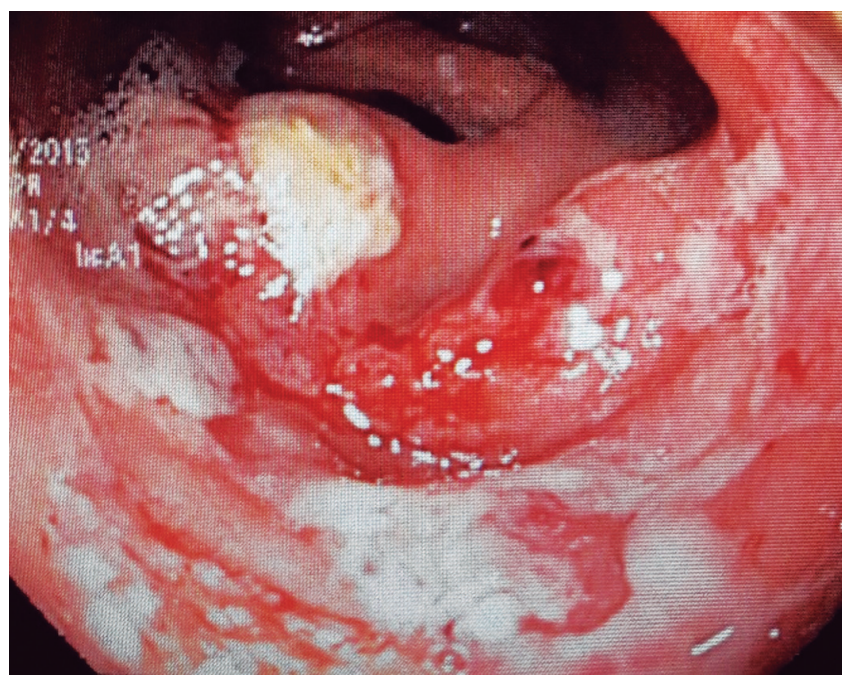

Figura 4. Colonoscopia en un paciente con VIH y diarrea sanguinolenta. En el ciego y colon derecho hay múltiples ulceraciones, mal definidas, con material necrótico en la superficie y fondo inflamatorio activo. En las biopsias se observó alteración estructural y trofozoítos de E. hystolitica con hemofagocitosis.

(38). Se ha estudiado el uso de cápsula endoscópica en pacientes con VIH y se encontraron anormalidades en el intestino delgado en el $89 \%$ de los casos (45). Por ahora no se ha evaluado su costo-efectividad y no se considera un examen de rutina.

En estudios radiológicos se han descrito algunos patrones como la tuberculosis que tiende a afectar región ileocecal, con engrosamiento del íleon y ciego, que simula la enfermedad de Crohn (46). La infección por MAC cursa con compromiso de yeyuno y engrosamiento de pliegues (3). En la infección por CMV, además de las úlceras en el colon, pueden encontrarse trombosis por vasculitis con isquemia y perforación de vísceras (3). El sarcoma de Kaposi puede afectar cualquier parte del aparato digestivo y se observa como lesiones largas y planas o submucosas asociadas con engrosamiento de pliegues (47). El linfoma no hodgkiniano (LNH) usualmente compromete el íleon terminal, por lo que se forman lesiones tipo masa y úlceras, con extensión del tumor al mesenterio y ganglios adyacentes (48) (Figura 2).

\section{Tercera etapa: evaluación de la TARV (34)}

Se ha descrito diarrea asociada con la TARV hasta en un $2 \%$ a $19 \%$ de los pacientes (49). Si se considera que hay relación entre un medicamento de la TARV y la diarrea, esta tercera etapa puede preceder a la segunda. Siempre se debe tener un concepto del infectólogo antes de considerar suspender o cambiar medicamentos de la TARV (Figura 2). 


\section{TRATAMIENTO DE LA DIARREA ASOCIADA CON ENTEROPATÍA POR VIH}

Lograr controlar la diarrea en este grupo de pacientes es importante: ayuda a mejorar la adherencia a la TARV, el estado nutricional, la estabilidad en el peso y la calidad de vida $(50,51)$. Inicialmente, se intenta mejorar los síntomas con modificaciones en el estilo de vida y, según la respuesta, se adicionan medicamentos. En pacientes con conteo bajo de CD4, la intervención con mayor efectividad será el inicio de la TARV.

\section{Manejo no farmacológico}

Un estudio con 75 pacientes comparó 2 grupos, uno con dieta convencional frente a uno con restricción de algunos alimentos (baja en grasas, fibra insoluble, cafeína, libre de lactosa y alta en fibra soluble). En los resultados se encontró que en el último grupo a las 24 semanas se presentó mejoría de la diarrea (52). En un ensayo clínico de 25 pacientes, el suplemento con L-glutamina demostró que podría reducir la severidad de la diarrea en comparación con placebo (53).

La evidencia del uso de probióticos es controversial. Un ensayo clínico de 17 pacientes con Lactobacillus GG por 2 semanas no pudo demostrar desenlaces positivos (54). Otro ensayo clínico con cepas de Lactobacillus durante 2 días resolvió la diarrea en 12/12 pacientes en comparación con $2 / 12$ pacientes que recibieron yogur sin probióticos (55). Un estudio de 69 pacientes con cepas de Lactobacillus por 25 semanas no demostró mejoría en síntomas relacionados con la diarrea (56). Con los resultados de estos estudios pequeños, el uso de probióticos no se puede recomendar.

\section{Manejo farmacológico}

\section{Agentes antimotilidad}

La loperamida y el difenoxilato buscan enlentecer el tránsito intestinal y aumentar la absorción de agua y sodio. Una revisión de Cochrane no demostró resultados positivos en diarrea asociada con VIH (57). Un estudio retrospectivo encontró que el $32 \%$ de los pacientes que recibían nelfinavir respondieron al uso de loperamida (58). Hay que tener en cuenta que el uso crónico de loperamida puede causar efectos adversos, incluida la interacción con inhibidores de proteasa. De todas maneras, el uso de este medicamento en VIH parece ser efectivo y seguro, y se debería utilizar como primera línea de tratamiento (50). La evidencia con difenoxilato es escasa y controversial (58). Este medicamento cruza la barrera hematoencefálica y tiene riesgo de abuso y dependencia. Se sugiere reservar para pacientes refractarios a otras medidas farmacológicas.

\section{Agentes antisecretores}

El octreótido de uso subcutáneo ha sido estudiado y la mayoría de estudios datan de la era pre-TARV. Algunos estudios encontraron una reducción en el volumen y frecuencia de las deposiciones $(59,60)$. Se deben tener en cuenta efectos adversos como hipoglucemia, formación de barro biliar, náuseas, dolor abdominal y constipación (50). Se debe reservar su uso en pacientes refractarios, evaluando riesgo/beneficio.

El crofelemer es un medicamento aprobado por la Food and Drug Administration (FDA) para la mejoría sintomática de la diarrea no infecciosa en pacientes con VIH. La dosis recomendada es de $125 \mathrm{mg} 2$ veces al día. Actúa de manera simultánea inhibiendo 2 canales de cloro: el CFTR (cystic fibrosis transmembrane conductance regulator) en las membranas apicales y el CaCC (calcium-activated chloride channel) en las membranas epiteliales, que disminuye la secreción de sodio y agua a la luz intestinal. Tiene absorción sistémica mínima, con acción directa a nivel intestinal (61), lo que le confiere poca interacción con otros medicamentos, con efecto clínico hacia las 4 semanas $(62,63)$.

El estudio ADVENT incluyó 374 pacientes con diarrea no infecciosa asociada con VIH, que persistía a pesar del manejo con loperamida (64). En su primera fase, demostró mejoría clínica con dosis de 125 y $500 \mathrm{mg}$ frente a placebo $(20,5 \%$ y $19,6 \%$ frente a $2 \%, p<0,0024)$. Se evaluó la seguridad a largo plazo en un estudio de fase III por 48 semanas en 250 pacientes; en los resultados se encontró 9,2\% de eventos adversos atribuidos al medicamento, sin cambios en el nivel de CD4+ ni de carga viral (65). En pacientes sin respuesta posterior a 3 meses, debe descontinuarse y probar otras alternativas. En nuestro medio, este medicamento ya tiene aprobación por el Instituto Nacional de Vigilancia de Medicamentos y Alimentos (INVIMA).

Para algunas otras terapias como suplementos de fibra, inmunoglobulina bovina, curcumina, sales de bismuto y racecadotril, la evidencia no es fuerte o los resultados no han sido positivos, por lo que no se recomiendan.

\section{CONCLUSIONES}

La diarrea en los pacientes con VIH es un síntoma común que puede causar deterioro en la calidad de vida, desnutrición e inclusive compromiso sistémico; por tanto, requiere de un abordaje y tratamiento adecuados. En el estudio diagnóstico siempre se deben considerar causas infecciosas, incluidos los gérmenes oportunistas. Como se demostró en nuestra revisión, se debe establecer el control inmunovirológico del paciente, ya que el abordaje sobre las posibles causas de la diarrea será más dirigido y específico. De igual manera, siempre debe evaluarse el impacto de la TARV 
sobre la diarrea en pacientes con VIH. Finalmente, si se considera que el paciente está en el contexto de enteropatía por VIH, el manejo se enfoca en buscar alivio sintomático, mejorar el estado nutricional y garantizar una adecuada adherencia a la TARV. Ya se cuenta con nuevos medicamentos como el crofelemer, con el que se espera mejorar la calidad de vida de los pacientes.

\section{REFERENCIAS}

1. Logan C, Beadsworth MB, Beeching NJ. HIV and diarrhoea: what is new? Curr Opin Infect Dis. 2016;29(5):486-94. doi: 10.1097/QCO.0000000000000305.

2. MacArthur RD, DuPont HL. Etiology and pharmacologic management of noninfectious diarrhea in HIV-infected individuals in the highly active antiretroviral therapy era. Clin Infect Dis. 2012;55(6):860-7. doi: 10.1093/cid/cis544.

3. Feasey NA, P. Healey P, Gordon MA. Review article: the aetiology, investigation and management of diarrhoea in the HIV-positive patient. Aliment Pharmacol Ther. 2011;34(6):587-603. doi: 10.1111/j.13652036.2011.04781.x.

4. Andrade FM. Quiroga A. Builes C. et al. Epidemiología de la infección por el virus de inmunodeficiencia humana en pacientes hospitalizados en una institución de alta complejidad y enseñanza universitaria en Medellín, Colombia. Infectio. 2016;20(1):9-16. doi: 10.1016/j. infect.2015.05.004.

5. Flórez AC, García DA, Moncada L, et al. Prevalencia de microsporidios y otros parásitos intestinales en pacientes con infección por VIH, Bogotá, 2001. Biomédica. 2003;23(3):274-82. doi: 10.7705/biomedica.v23i3.1221.

6. Shah S, Kongre V, Kumar V, et al. A study of parasitic and bacterial pathogens associated with diarrhea in HIV-positive patients. Cureus. 2016;8(9):e807. doi: 10.7759/cureus.807.

7. Oude Munnink BB, Canuti M, Deijs M, et al. Unexplained diarrhoea in HIV-1 infected individuals. BMC Infect Dis. 2014;14:22. doi: 10.1186/1471-2334-14-22.

8. Krones E, Högenauer $\mathrm{Ch}$. Diarrhea in the immunocompromised patient. Gastroenterol Clin North Am. 2012;41(3):677-701. doi: 10.1016/j.gtc.2012.06.009.

9. Fernández-Cruz A, Muñoz P, Mohedano R, et al. Campylobacter bacteremia: clinical characteristics, incidence, and outcome over 23 years. Medicine (Baltimore). 2010;89(5):319-30. doi: 10.1097/MD.0b013e3181f2638d.

10. Hohmann EL. Nontyphoidal salmonellosis. Clin Infect Dis. 2001;32(2):263-9. doi: 10.1086/318457.

11. Wilcox CM. Gastrointestinal consequences of infection with human immunodeficiency virus. En: Feldman M, Friedman LS, Brandt LJ (editores). Sleisenger and Fordtrans's gastrointestinal and liver disease. Filadelfia: Elsevier Saunders; 2010. pp. 526-30.

12. Burgers WA, Riou C, Mlotshwa M, et al. Association of HIV-specific and total CD8+ $\mathrm{T}$ memory phenotypes in subtype C HIV-1 infection with viral set point. J Immunol. 2009;182(8):4751-61. doi: 10.4049/jimmunol.0803801.
13. Wallace JM, Hannah JB. Mycobacterium avium complex infection in patients with the acquired immunodeficiency syndrome. A clinicopathologic study. Chest. 1988;93(5):926-32. doi: 10.1378/chest.93.5.926.

14. Gordin FM, Cohn DL, Sullam PM, et al. Early manifestations of disseminated Mycobacterium avium complex disease: a prospective evaluation. J Infect Dis. 1997;176(1):126-32. doi: $10.1086 / 514014$.

15. Bhaijee F, Subramony C, Tang SJ, et al. Human immunodeficiency virus associated gastrointestinal disease: common endoscopic biopsy diagnoses. Patholog Res Int. 2011;2011:247923. doi: 10.4061/2011/247923.

16. Gordon SN, Cervasi B, Odorizzi P, et al. Disruption of intestinal CD4 $1 \mathrm{~T}$ cell homeostasis is a key marker of systemic CD4 $1 \mathrm{~T}$ cell activation in HIVinfected individuals. J Immunol 2010;185(9):5169-79. doi: 10.4049/jimmunol.1001801.

17. Loo VG, Bourgault AM, Poirier L, et al. Host and pathogen factors for Clostridium difficile infection and colonization. N Engl J Med. 2011;365(18):1693-703. doi: 10.1056/ NEJMoa1012413.

18. Deshpande A, Pasupuleti V, Rolston DD, et al. Diagnostic accuracy of real-time polymerase chain reaction in detection of Clostridium difficile in the stool samples of patients with suspected Clostridium difficile infection: a meta-analysis. Clin Infect Dis. 2011;53(7):e81-90. doi: 10.1093/cid/ cir505.

19. Louie TJ, Miller MA, Mullane KM, et al. Fidaxomicin versus vancomycin for Clostridium difficile infection. N EnglJ Med. 2011;364(5):422-31. doi: 10.1056/NEJMoa0910812.

20. Lawlor G, Moss AC. Cytomegalovirus in inflammatory bowel disease: pathogen or innocent bystander? Inflamm Bowel Dis. 2010;16(9):1620-7. doi: 10.1002/ibd.21275.

21. Ljungman P, Griffiths P, Paya C. Definitions of cytomegalovirus infection and disease in transplant recipients. Clin Infect Dis. 2002;34(8):1094-7. doi: 10.1086/339329.

22. Huston CD. Intestinal protozoa. En: Feldman M, Friedman LS, Brandt LJ (editores). Sleisenger and Fordtrans's gastrointestinal and liver disease. Filadelfia: Elsevier Saunders; 2010. pp. 1914-8.

23. Ma P. Cryptosporidiosis and immune enteropathy: a review. Curr Clin Top Infect Dis. 1987;8:99-153.

24. Rossignol JF. Nitazoxanide in the treatment of acquired immune deficiency syndrome-related cryptosporidiosis: results of the United States compassionate use program in 365 patients. Aliment Pharmacol Ther 2006;24(5):887-94. doi: 10.1111/j.1365-2036.2006.03033.x.

25. Silva CV, Ferreira MS, Borges AS, et al. Intestinal parasitic infections in HIV/AIDS patients: experience at a teaching hospital in central Brazil. Scand J Infect Dis. 2005;37(3):211-5. doi: 10.1080/00365540410020875.

26. Concha R, Harrington W Jr, Rogers AI. Intestinal strongyloidiasis: recognition, management, and determinants of outcome. J Clin Gastroenterol. 2005;39(3):203-11. doi: 10.1097/01.mcg.0000152779.68900.33. 
27. Segarra-Newnham M. Manifestations, diagnosis, and treatment of Strongyloides stercoralis infection. Ann Pharmacother. 2007;41(12):1992-2001. doi: 10.1345/ aph.1K302.

28. Casotti JA, Motta TQ, Ferreira CU Jr, et al. Disseminated histoplasmosis in HIV positive patients in Espirito Santo state, Brazil: a clinical-laboratory study of 12 cases (19992001). Braz J Infect Dis. 2006;10(5):327-30. doi: 10.1590/ S1413-86702006000500005.

29. Assi M, McKinsey DS, Driks MR, et al. Gastrointestinal histoplasmosis in the acquired immunodeficiency syndrome: report of 18 cases and literature review. Diagn Microbiol Infect Dis. 2006;55(3):195-201. doi: 10.1016/j. diagmicrobio.2006.01.015.

30. Kartalija M, Sande MA. Diarrhea and AIDS in the era of highly active antiretroviral therapy. Clin Infect Dis 1999;28(4):701-5. doi: 10.1086/515191.

31. Cello JP, Day LW. Idiopathic AIDS enteropathy and treatment of gastrointestinal opportunistic pathogens. Gastroenterology. 2009;136(6):1952-65. doi: 10.1053/j. gastro.2008.12.073.

32. O'Brien ME, Clark RA, Besch CL, et al. Patterns and correlates of discontinuation of the initial HAART regimen in an urban outpatient cohort. J Acquir Immune Defic Syndr. 2003;34(4):407-14. doi: 10.1097/00126334-200312010-00008.

33. Wu X, Sun L, Zha W, et al. HIV protease inhibitors induce endoplasmic reticulum stress and disrupt barrier integrity in intestinal epithelial cells. Gastroenterology. 2010;138(1):197-209. doi: 10.1053/j.gastro.2009.08.054.

34. Macarthur RD. Management of noninfectious diarrhea associated with HIV and highly active antiretroviral therapy. Am J Manag Care. 2013;19(12 Suppl):s238-45.

35. National Institute of Allergy and Infectious Diseases. Division of AIDS table for grading the severity of adult and pediatric adverse events. US Department of Health and Human Services [internet] 2014 [acceso el 11 de noviembre de 2017]. Disponible en: http://rsc.tech-res.com/ docs/default-source/safety/daids_ae_grading_table_v2_ nov2014.pdf.

36. Public Health England. UK Standards for Microbiology: investigation of specimens other than blood for parasites. NHS [internet] 2017 [acceso el 11 de noviembre de 2017]. Disponible en: https://www.gov.uk/government/ uploads/system/uploads/attachment_data/file/622944/ B_31i5.1.pdf.

37. American Society for Gastrointestinal Endoscopy. The role of endoscopy in the management of patients with diarrhea. Gastrointest Endosc. 2010;71(6):887-92. doi: 10.1016/j. gie.2009.11.025.

38. Cohen J, West $A B$, Bini EJ. Infectious diarrhea in human immunodeficiency virus. Gastroenterol Clin North Am. 2001;30(3):637-64. doi: 10.1016/S08898553(05)70203-X.

39. Olmos MA, Losso M, Ruvinsky S, et al. Decision analysis: diagnostic approach in HIV infection associated chronic diarrea. Acta Gastroenterol Latinoam. 2005;35(3):155-61.
40. Wilcox M, Schwartz D, Cotsonis G, et al. Chronic unexplained diarrhea in human immunodeficiency virus infection: determination of the best diagnostic approach. Gastroenterology. 1996;110(1):30-7. doi: 10.1053/ gast.1996.v110.pm8536874.

41. Kearney DJ, Steuerwald M, Koch J, et al. A prospective study of endoscopy in HIV -associated diarrea. Am J Gastroenterol. 1999;94(3):596-602. doi: 10.1111/j.15720241.1999.00920.x.

42. Wei SC, Hung CC, Chen MY, et al. Endoscopy in acquired immunodeficiency syndrome patients with diarrhea and negative stool studies. Gastrointest Endosc. 2000;51(4 Pt 1):427. doi: 10.1016/S0016-5107(00)70443-3.

43. Orenstein JM, Dieterich DT. The histopathology of 103 consecutive colonoscopy biopsies from 82 symptomatic patients with acquired immunodeficiency syndrome. Arch Pathol Lab Med. 2001;125(8):1042-6. doi: 10.1043/0003-9985(2001)125<1042:THOCCB>2.0.CO;2.

44. Bini EJ, Cohen J. Diagnostic yield and cost-effectiveness of endoscopy in chronic human immunodeficiency virus-related diarrea. Gastrointest Endosc. 1998;48(4):354-61. doi: 10.1016/S0016-5107(98)70003-3.

45. Oette M, Stelzer A, Göbels K, et al. Wireless capsule endoscopy for the detection of small bowel Diseases in HIV-1infected patients. Eur J Med Res. 2009;14(5):191-4.

46. Burrill J, Williams CJ, Bain G, et al. Tuberculosis: a radiologic review. Radiographics. 2007;27(5):1255-73. doi: $10.1148 / \mathrm{rg} .275065176$.

47. Restrepo CS, Martinez S, Lemos JA, et al. Imaging manifestations of Kaposi sarcoma. Radiographics. 2006;26(4):116985. doi: $10.1148 /$ rg.264055129.

48. Ghai S, Pattison J, O’Malley ME, et al. Primary gastrointestinal lymphoma: spectrum of imaging findings with pathologic correlation. Radiographics. 2007;27(5):1371-88. doi: $10.1148 / \mathrm{rg} .275065151$.

49. Hill A, Balkin A. Risk factors for gastrointestinal adverse events in HIV treated and untreated patients. AIDS Rev. 2009; $11(1): 30-8$.

50. Clay PG, Crutchley RD. Noninfectious diarrhea in HIV seropositive individuals: a review of prevalence rates, etiology, and management in the era of combination antiretroviral therapy. Infect Dis Ther. 2014;3(2):103-22. doi: 10.1007/s40121-014-0047-5.

51. Mangili A, Murman DH, Zampini AM, et al. Nutrition and HIV infection: review of weight loss and wasting in the era of highly active antirretroviral therapy from the nutrition for healthy living cohort. Clin Infect Dis. 2006;42(6):836-42. doi: 10.1086/500398.

52. Anastasi JK, Capili B, Kim AG, et al. Symptom management of HIV related diarrhea by using normal foods: a randomized controlled clinical trial. J Assoc Nurses AIDS Care. 2006;17(2):47-57. doi: 10.1016/j.jana.2006.01.005.

53. Huffman FG, Walgren ME. L-glutamine supplementation improves nelfinavir-associated diarrhea in HIV-infected individuals. HIV Clin Trials. 2003;4(5):324-9. doi: 10.1310/BFDT-J2GH-27L7-905G. 
54. Anukam KC, Osazuwa EO, Osadolor HB, et al. Yogurt containing probiotic Lactobacillus rhamnosus GR-1 and $\mathrm{L}$. reuteri $\mathrm{RC}-14$ helps resolve moderate diarrhea and increases CD4 count in HIV/ AIDS patients. J Clin Gastroenterol. 2008;42(3):239-43. doi: 10.1097/ MCG.0b013e31802c7465.

55. Salminen MK, Tynkkynen S, Rautelin H, et al. The efficacy and safety of probiotic Lactobacillus rhamnosus GG on prolonged, noninfectious diarrhea in HIV patients on antiretroviral therapy: a randomized, placebo-controlled, crossover study. HIV Clin Trials. 2004;5(4):183-91. doi: 10.1310/6F83-N39Q-9PPP-LMVV.

56. Hummelen R, Changalucha J, Butamanya NL, et al. Effect of 25 weeks probiotic supplementation on immune function of HIV patients. Gut Microbes. 2011;2(2):80-5. doi: 10.4161/gmic.2.2.15787.

57. Nwachukwu CE, Okebe JU. Antimotility agents for chronic diarrhoea in people with HIV/AIDS. Cochrane Database Syst Rev. 2008;(4):CD005644. doi: 10.1002/14651858. CD005644.pub2.

58. Sherman DS, Fish DN. Management of protease inhibitorassociated diarrhea. Clin Infect Dis. 2000;30(6):908-14. doi: $10.1086 / 313826$.

59. Cello JP, Grendell JH, Basuk P, et al. Effect of octreotide on refractory AIDS-associated diarrhea. A prospective, multicenter clinical trial. Ann Intern Med. 1991;115(9):705-10. doi: 10.7326/0003-4819-115-9-705.
60. Beaugerie L, Baumer P, Chaussade S, et al. Treatment of refractory diarrhoea in AIDS with acetorphan and octreotide: a randomized crossover study. Eur J Gastroenterol Hepatol. 1996;8(5):485-9.

61. CastroJG, Chin-BeckfordN.Crofelemerfor the symptomatic relief of non-infectious diarrhea in adult patients with HIV/ AIDS on anti-retroviral therapy. Expert Rev Clin Pharmacol. 2015;8(6):683-90. doi: 10.1586/17512433.2015.1082424.

62. Leonard C, Chordia P, MacArthur RD. Profile of crofelemer for the symptomatic treatment of diarrhea in HIV-infected persons. Botanics Target Ther. 2015;5:21-5. doi: 10.2147/ BTAT.S42267.

63. Crutchley RD, Miller J, Garey KW. Crofelemer, a novel agent for treatment of secretory diarrhea. Ann Pharmacother. 2010;44(5):878-84. doi: 10.1345/aph.1M658.

64. Macarthur RD, Hawkins TN, Brown SJ, et al. Efficacy and safety of crofelemer for noninfectious diarrhea in HIVseropositive individuals (ADVENT trial): a randomized, double-blind, placebo-controlled, two-stage study. HIV Clin Trials. 2013;14(6):261-73. doi: 10.1310/hct1406-261.

65. Hawkins T, MacArthur RD, Brown SJ, et al. Safety and tolerability of crofelemer $125 \mathrm{mg}$ twice daily in the treatment of noninfectious diarrhea in HIV-seropositive patients on antiretroviral therapy: results from a Phase 3, 48-week openlabel study. Meeting of the Infectious Diseases Society of America. 2013. 